\title{
Optimizing Regional Therapy for Melanoma
}

\author{
Georgia M. Beasley, MD, and Douglas S. Tyler, MD \\ Department of Surgery, Duke University Medical Center, Durham, NC 27710
}

In this issue of Annals of Surgical Oncology, Kroon et al. describe the toxicity of isolated limb infusion with melphalan and actinomycin D in their experience with 185 patients over 15 years. ${ }^{1}$ This work continues to expand on the group's notable contributions to regional chemotherapeutics for extremity melanoma including their initial description and utilization of isolated limb infusion (ILI) in the early 1990s. ${ }^{2}$ ILI has been explored as a minimally invasive alternative to hyperthermic isolated limb perfusion (HILP) for advanced extremity melanoma whereby placement of percutaneously placed catheters allows delivery of regional chemotherapy to an isolated limb. In the current study, ILI was found to be a safe alternative to HILP with less long-term morbidity than HILP. Previously, the Sydney Melanoma Group has reported response rates that are less than traditional response rates to HILP but certainly not outside the range of reported studies of HILP. ${ }^{3}$ Due to the safety and efficacy of the treatment as well as disappointing results of a multicenter HILP trial, ${ }^{4}$ ILI is now utilized at several major US centers and has been demonstrated to be a well-tolerated treatment alternative for patients with advanced extremity melanoma.,

As with any new regional treatment, the goal should be to define the procedure's therapeutic index, which balances the treatment's toxicity with its ability to control disease. Results of the current study suggest that there may be additional methods to reduce toxicity from ILI by altering modifiable factors which were found to be predictive of higher grades of toxicity. In addition this study's results further support a role for ILI as a safe treatment in the armamentarium of therapeutic options for patients with advanced extremity melanoma.

ILI is essentially a low-flow HILP performed by insertion of percutaneous catheters in the contralateral limb, in

(C) Society of Surgical Oncology 2009

Published Online: 3 February 2009

D. S. Tyler, MD

e-mail: tyler002@acpub.duke.edu contrast to HILP where open surgical cannulation of the artery and vein are performed. ILI is performed attempting to achieve temperatures in the range $37-38.5^{\circ} \mathrm{C}$ and the blood is not oxygenated, leading to hypoxia and acidosis in the limb. The hypoxia and acidosis of ILI have previously been suggested to be beneficial in terms of response. ${ }^{7}$ In HILP, the catheters are connected to a cardiopulmonary bypass circuit which maintains physiologic oxygenation and $\mathrm{pH}$. Temperatures in HILP generally range from $38.5^{\circ} \mathrm{C}$ to $40^{\circ} \mathrm{C}$. While hyperthermia is well documented to increase the cytotoxicity induced by melphalan, ${ }^{8}$ excessive heat can also increase the toxicity of melphalan to normal tissue. ILI also differs from HILP in that ILI uses less melphalan per liter of extremity treated, circulates blood in an isolated extremity at a much slower rate than HILP (50$100 \mathrm{~mL} / \mathrm{min}$ in $\mathrm{ILI}^{7}$ versus $350-1,000 \mathrm{~mL} / \mathrm{min}$ in $\mathrm{HILP}^{9}$ ), and treatment is only for $30 \mathrm{~min}$ as compared with $60 \mathrm{~min}$ in HILP. These differences in the method of drug delivery are important to consider when comparing toxicity and response between the two procedures.

In both ILI and HILP, there appears to be no relationship between significant limb toxicity and response to treatment. ${ }^{7,10,11}$ As such, minimizing toxicity from these procedures as discussed by the authors should be a goal of therapy. In the current study, high-grade limb toxicity (Wiberdink toxicity $\geq$ grade 3 ) occurred in $42 \%(n=77)$ of patients. Only $3 \%(n=5)$ of patients had grade IV toxicities and there were no grade $\mathrm{V}$ toxicities (grade $\mathrm{V}$ toxicity is a toxicity necessitating amputation). Systemic toxicities were minimal and melphalan was detected in the systemic circulation in only $6 \%$ of patients. There were no systemic effects clearly associated with leakage of the small amounts of melphalan into the systemic circulation. Low circulating volumes seen in ILI were not related to increased limb toxicity. Overall toxicity from this study is in a similar range to the spectrum of toxicity after HILP. However, as the authors point out, long-term morbidity and severe toxicity (grade V) appear to be less with ILI. In a 
retrospective comparison of patients undergoing HILP and ILI at Duke University, patients undergoing ILI had significantly less toxicity than those undergoing HILP. ${ }^{5}$ In a recent retrospective US multicenter study of ILI, there was one treatment-associated amputation in 162 patients. ${ }^{11}$ Analysis of data from the SMU, Duke University, MD Anderson Cancer Center, and other centers performing ILI in the USA demonstrated the rate of toxicity necessitating amputation to be approximately five- to tenfold less in patients undergoing ILI compared with HILP $(0.2-0.3 \%$ in ILI versus $1-2 \%$ in HILP). ${ }^{10}$ The results of the current study are thus in accordance with other reports and suggest that ILI can be performed safely with less severe and less long-term morbidity than HILP.

The authors performed a detailed analysis of factors that might predict toxicity, which may be important in minimizing toxicity from ILI. Interestingly, on univariate analysis the authors found high peak and high final melphalan concentrations as well as increased serum creatine phosphokinase (CK) postoperatively to be predictive of grade III/IV toxicity. In addition, on multivariate analysis both higher melphalan concentration and shorter tourniquet time were independent factors for developing grade III/IV toxicity. Higher peak melphalan concentration has shown to be predictive of toxicity in HILP ${ }^{12}$ while higher melphalan dose has been associated with increased toxicity in a multicenter study of ILI. $^{5}$ Based on pharmacokinetic analysis at our own institution, ${ }^{13,14}$ which demonstrated wide variability in melphalan concentration in patients undergoing ILI, we began modifying the melphalan dose for ideal body weight (IBW) ${ }^{11}$ In both our series of ILI and in a recent multicenter study of ILI, correction of the melphalan dose for IBW significantly reduced toxicity without altering complete response rates. In a similar fashion, a melphalan dose corrected for IBW was also found to be associated with significantly lower postoperative peak CK levels in those two studies. ${ }^{5,11}$ Consistent with the SMU data, the recent multi-institutional study of ILI also found higher peak CK to be significantly associated with higher grades of toxicity. Correction of the melphalan dose for IBW is thus a method of lowering the melphalan dose which successfully decreases postoperative $\mathrm{CK}$ values and minimizes limb toxicity without altering complete response $(\mathrm{CR})$ rates. This dosing modification also appears to decrease toxicity in HILP without altering CR rates. ${ }^{13}$

In addition to minimizing toxicity, another goal of regional therapy is to maximize the complete response rate. Complete responses are the most important outcome measurement with the greatest potential therapeutic benefit as most patients with partial responses, stable disease, or progressive disease require additional treatment. While dose reductions seem to decrease overall response rates, no difference in CR rates have been noted. Additional strategies to increase CR rates have recently been explored; for example, a recent phase I trial using the targeted agent ADH-1, which disrupts $\mathrm{N}$-cadherin binding, was associated with a $50 \% \mathrm{CR}$ rate when given systemically $6-10 \mathrm{~h}$ before melphalan-based treatment. ${ }^{14,15}$ The multicenter phase II trial of systemic ADH-1 and regional ILI has completed accrual and is currently under analysis. Other targeted compounds currently in phase I trials being studied for their ability to increase CR rates include Sorafenib and buthionine sulfoximine (BSO), which are given systemically in combination with ILI treatment.

While the exact place of ILI in managing regionally advanced disease remains to be determined, the work of the SMU group, which is now being replicated in other centers in the USA, would argue that ILI might be the best initial therapy given its low toxicity and approximate $30-40 \%$ CR rate. Patients who fail ILI could then be treated with HILP, repeat ILI, or protocol-based ILI. One challenge for the regional chemotherapy group of surgeons is to utilize studies such as the current one to define the current "standard" approach to ILI in an attempt to establish a consensus statement or methodology on the treatment variables associated with the procedure. Achieving agreement on such variables as melphalan dosing, target tourniquet times, response determinations, and use of papaverine would not only allow for more uniform implementation of the therapy but would also help develop a basis upon which incremental advances could more readily be achieved.

ACKNOWLEDGEMENTS Dr. Tyler is co-inventor on a patent entitled "Cancer treatment methods using cadherin antagonists in combination with anticancer agents." The patent application number is $60 / 848,624$ and this patent was filed on $9 / 27 / 06$. Dr. Tyler has signed over all rights to the patent to the Federal Government. Dr. Tyler has received some research funding from Adherex Technologies Inc. for his laboratory. Dr. Tyler also has material transfer agreements with Schering, Genta, Inotek, and Bayer to provide his laboratory with reagents to study. Dr. Tyler does not receive grant support from these companies.

\section{REFERENCES}

1. Kroon HM, Moncrieff M, Kam PCA, et al. Factors predictive of acute regional toxicity after isolated limb infusion with melphalan and actinomycin D in melanoma patients. Ann Surg Oncol. 2009:16 (in press).

2. Thompson JF, Waugh RC, Saw RPM, Kam PCA. Isolated limb infusion with melphalan for recurrent limb melanoma: a simple alternative to isolated limb perfusion. Reg Cancer Treat. 1994; 7:188-92.

3. Kroon HM, Moncrieff M, Kam PCA. Outcomes following isolated limb infusion for melanoma. A 14-year experience. Ann Surg Oncol. 2008;15(11):3003-13; epub 2008 May 29.

4. Cornett WR, McCall LM, Petersen RP, Ross MI, Briele HA, Noyes RD, et al. Prospective randomized multicenter trial of hyperthermic isolated limb perfusion with melphalan alone versus melphalan plus tumor necrosis factor: American College of 
Surgeons Oncology Group Trial Z0020. J Clin Oncol. 2006;24:4196-201.

5. Beasley GM, Petersen RP, McMahon NS, Mosca PJ, Zager JS, Hochwald SN, et al. A multi-institutional experience of isolated limb infusion: defining response and toxicity in the United States. J Am Coll Surg. 2008 (accepted).

6. Brady MS, Brown K, Patel A, Fisher C, Marx W. A phase II trial of isolated limb infusion with melphalan and dactinomycin for regional melanoma and soft tissue sarcoma of the extremity. Ann Surg Oncol. 2006;13(8):1123-29.

7. Lindner P, Doubrovsky A, Kam PCA, Thompson JF. Prognostic factors after isolated limb infusion with cytotoxic agents for melanoma. Ann Surg Oncol. 2002;9:127-36.

8. Storm FK, Morton DL. Value of therapeutic hyperthermic limb perfusion in advanced recurrent melanoma of the lower extremity. Am J Surg. 1985;15:32-5.

9. Koops HS, Lejeune KJ, Kroon BBR, Hoekstra HJ. Isolated limb perfusion for melanoma: technical aspects. In: Thompson JF, Morton DL, Kroon BBR, editors. Textbook of melanoma. London: Martin Dunitz; 2004. p. 404-9.

10. Vrouenraets BC, Hart GA, Eggermont AM, Klaase JM, van Geel $\mathrm{BN}$, Nieweg OE, et al. Relation between limb toxicity and treatment outcomes after isolated limb perfusion for recurrent melanoma. J Am Coll Surg. 1999;188:522-30.
11. Beasley GM, Petersen RP, Yoo JS, Aloia T, Petros W, Sanders G, et al. Isolated limb infusion for in-transit malignant melanoma of the extremity: a well tolerated but less effective alternative to hyperthermic isolated limb perfusion. Ann Surg Oncol. 2008;15(8):2195-205; epub 2008 June.

12. Thompson JF, Kam PCA, Ramzan I, Yau DF. Clinical pharmacokinetics of melphalan during isolated limb perfusion: compartmental modeling and moment analysis. Reg Cancer Treat. 1995;8:83-7.

13. Cheng T-Y, Grubbs E, Abdul-Wahab O, Leu SY, Hung CF, Petros W, et al. Marked variability of melphalan plasma drug levels during regional hyperthermic isolated limb perfusion. Am J Surg. 2003;186(5):460-7.

14. McMahon N, Cheng TY, Beasley GM, et al. Optimizing melphalan pharmacokinetics in regional melanoma therapy: does correcting for ideal body weight alter regional response or toxicity? Ann Surg Oncol. 2008 (accepted).

15. Beasley GM, McMahon N, Sanders G, et al. A phase I/II study of systemic ADH-1 in combination with isolated limb infusion with melphalan in patients with locally advanced in-transit melanoma. American Society of Clinical Oncology (ASCO) 44th annual meeting. May 30-June 3, 2008. Chicago, IL. 